\section{Watchful waiting is a reasonable option for men with minimally symptomatic inguinal hernia}

Surgical repair of an inguinal hernia can alleviate pain and prevent a HERNIA ACCIDENT, although such surgery is not risk-free. Many men without troublesome symptoms, therefore, delay surgery until their hernia symptoms escalate. A multicenter, randomized, US trial compared this treatment strategy with routine surgery, and concluded that watchful waiting is safe and acceptable for men with minimal hernia symptoms.

Hernia accidents were rare in this trial (1.8 per 1,000 patient-years), although most participants were in the 40-65 age-group (mean age 57.5) and other studies suggest that most hernia accidents occur in elderly patients. Men assigned to watchful waiting whose symptoms worsened, and who consequently requested surgery, had no greater risk of complications from the procedure than men who underwent prophylactic repair.

Men assigned to watchful waiting who crossed over to surgery had reported high baseline levels of sensory and affective pain during their daily activities, and impaired physical functioning. Crossover patients reported a greater improvement in such parameters after surgery compared with men who underwent prophylactic surgical repair; the authors suggest that these characteristics might, therefore, help physicians select an appropriate treatment strategy.

The main limitation of the study was its short, median 3.2-year duration. The risk of a hernia accident increases with the length of time the hernia is present, so the authors established a voluntary long-term registry for all enrolled patients. Their ongoing annual assessment of patient-reported outcomes includes hernia accidents, pain, and recurrence.

Caroline Barranco

Original article Fitzgibbons RJ Jr et al. (2006) Watchful waiting vs repair of inguinal hernia in minimally symptomatic men: a randomized clinical trial. JAMA 295: 285-292

\section{Vaccination prevents perinatal transmission of hepatitis B virus}

EBM Perinatal mother-to-child transmission accounts for $\sim 50 \%$ of all new hepatitis $B$ infections. The risk of transmission depends on the mother's antigen status - the highest transmission rates are seen in mothers who are positive for both hepatitis $B$ surface antigen and $e$ antigen. A plasma-derived and a recombinant hepatitis $B$ vaccine are available; both require repeated injections to induce adequate protection. Immunoglobulin provides immediate but short-term protection.

A systematic review and meta-analysis of 26 randomized, controlled trials showed that immunizing the infants of mothers who were positive for hepatitis $B$ surface antigen prevented hepatitis B infection effectively. Both vaccines had similar efficacy, although the combination of a vaccine plus immunoglobulin was more effective than either vaccine alone. The efficacy of immunization did not correlate with the mother's e-antigen status or the vaccination schedule used, although the authors note that few infants were included.

There were insufficient data to show that vaccination benefited children of mothers who were negative for e antigen (transmission rates are, in general, markedly lower in this group, compared with mothers positive for both antigens). Infants born to such mothers are often asymptomatic and only transiently infected. Most trials did not adequately report adverse events, but the available data indicated that immunization was safe.

Recombinant vaccine gave higher antibody titers to hepatitis $B$ surface antigen than plasma-derived vaccine, and its use might reduce the inadvertent transmission of other blood-borne infections. Optimal dose and vaccination schedules remain to be determined.

Caroline Barranco

Original article Lee C et al. (2006) Effect of hepatitis B immunization in newborn infants of mothers positive for hepatitis B surface antigen: systematic review and metaanalysis. BMJ 332: $328-336$

\section{Accurate detection of cirrhosis by transient elastography}

Progressive hepatic fibrosis and cirrhosis are common features of most chronic liver diseases, and can lead to a number of complications including liver failure and portal hypertension. The current gold standard for assessing fibrosis is liver biopsy; however, a novel, noninvasive method has been developed-transient elastography - that can provide accurate measure-

\section{GLOSSARY}

HERNIA ACCIDENT

Acute incarceration

of a hernia, with bowel obstruction and/or strangulation of intraabdominal contents; can be life-threatening 